\title{
A study on self-similar surfaces
}

\author{
Mustafa Altın ${ }^{\mathrm{a}, *}$, Müge Karadağg ${ }^{\mathrm{b}}$, H.Bayram Karadağ ${ }^{\mathrm{b}}$ \\ ${ }^{a}$ Bingöl University, Vocational School of Technical Sciences, 12000, Bingöl, Turkey. \\ bInönü University, Faculty of Art and Science, Department of Mathematics,44280, Malatya, Turkey.
}

\begin{abstract}
In this paper, we study the self-similar surfaces in 4-dimensional Euclidean space $\mathbb{E}^{4}$. We give an if and only if condition for a generalized rotational surfaces in $\mathbb{E}^{4}$ to be self-similar. In addition we examine self-similarity of some special surfaces in $\mathbb{E}^{4}$. Furthermore we investigate the self-similar condition of Tensor Product surfaces and Meridian surfaces in $\mathbb{E}^{4}$.
\end{abstract}

Keywords: Self similar surface, tensor product surfaces, generalized rotating surfaces, meridian surface.

2010 MSC: 47H10, 54H25.

(C)2019 All rights reserved.

\section{Introduction}

The theory of curves and surfaces in Euclidean space is very important notion in differential geometry. There are many applications of curve and surfaces, such as computer engineering, physics and linear equations. Especially, curvature properties are used for obtaining textural features in pattern recognition and image segmentation [14,15]. On the other hand, some special surfaces in $\mathbb{E}^{4}$ such as generalized rotating surfaces [10], Lawson surfaces [16], Aminow surfaces [1], Vranceanu surfaces [20], flat Klein bottle [19], Banchoff surfaces [6], Loop surfaces [12], tensor product surfaces [17], meridian surfaces [11] are important the differential geometry of surfaces.

Gradient flow of area functional on submanifolds n-dimensional of a Riemannian manifold is called the Mean Curvature Flow (MCF). To take more information more about the behavior of the MCF, selfshrinkers have been studied. Many authors study on self-shrinking surfaces to elaborate the regularity of the MCF. This studies become important to work the self-similar curves and surfaces. In literature there are several works on self-similar curves and surfaces [2-4,9].

In this paper, firstly we present some fundamental facts on self-similar surfaces then we prove necessary and sufficient conditions for generalized rotating surfaces, meridian surfaces and tensor product surfaces in $\mathbb{E}^{4}$ to be self-similar. In addition, we examine some important special surfaces such as Lawson surface, Aminow surface, Vranceanu surfaces, flat Klein bottle surfaces, Banchoff surfaces and Loop surfaces are self-similar surfaces or not.

\footnotetext{
*Corresponding author

Email addresses: maltin@bingol.edu.tr (Mustafa Altın), muge.karadag@inonu.edu.tr (Müge Karadağ),

bayram.karadag@inonu.edu.tr (H.Bayram Karadağ)
}

doi: $10.22436 / \mathrm{mns} .04 .01 .04$

Received: 2018-12-18 Revised: 2019-01-09 Accepted: 2019-02-30 


\section{Preliminaries}

In this section we give a short survey for surface theory.

Let $S \subset \mathbb{E}^{4}, \mathrm{U} \subset \mathrm{S}$ be a neighborhood of a point in $S$ and $\mathrm{V} \subset \mathbb{E}^{2}$. If $X: \mathrm{V} \longrightarrow \mathbb{E}^{4}$ has the following conditions then it is called smooth 2-surface in $\mathbb{E}^{4}$ :

i) $\mathrm{X}: \mathrm{V} \longrightarrow \mathrm{U}$ is a homeomorphism;

ii) $X(u, v)=(x(u, v), y(u, v), z(u, v))$ has derivatives of all orders;

iii) at all points, the first derivatives $x_{\mathfrak{u}}=\frac{\partial x}{\partial u}$ and $x_{v}=\frac{\partial x}{\partial v}$ are linearly independent [13].

Now we recall some fundamental equations for surfaces in $n$ - dimensional Euclidean space.

Let $M$ be a surface which is given by $X(u, v)$. The coefficients of the first fundamental form of a $X(u, v)$ are given by

$$
E=\left\langle X_{u}, X_{u}\right\rangle, F=\left\langle X_{v}, X_{u}\right\rangle, G=\left\langle X_{v}, X_{v}\right\rangle,
$$

where $\langle$,$\rangle is standard inner product in \mathbb{E}^{4}$. If $W=E G-F^{2} \neq 0$, then surface $X(u, v)$ is regular.

Let $T_{P} M$ and $T_{P}^{\perp} M$ be tangent space and normal space of $M$ in $\mathbb{E}^{n}$ at the point $P$, and $\widetilde{\nabla}$ and $\nabla$ be Riemannian and normal connections in the tangent bundle $\chi(M)$ and normal bundle $\chi^{\perp}(M)$, respectively. Since $T_{P}^{\perp} M$ is orthogonal component of $T_{p} M$ in $\mathbb{E}^{n}$, for $X_{1}, X_{2}$ tangent vector fields of $M$ we have

$$
\nabla_{\mathrm{X}_{1}} \mathrm{X}_{2}=\left(\widetilde{\nabla}_{\mathrm{X}_{1}} \mathrm{X}_{2}\right)^{\mathrm{T}}
$$

The second fundamental form of $X(u, v)$ is given by

$$
h: \chi(M) \times \chi(M) \rightarrow \chi^{\perp}(M), \quad h\left(X_{i}, X_{j}\right)=\widetilde{\nabla} X_{i} X_{j}-\nabla X_{i} X_{j},(1 \leqslant i, j \leqslant 2) .
$$

where

$$
\nabla_{X_{i}} X_{j}=\sum_{k=1}^{2} \Gamma_{i j}^{k} X_{k},(1 \leqslant i, j \leqslant 2), \quad h\left(X_{i}, X_{j}\right)=\sum_{k=1}^{n-2} c_{i j}^{k} N_{k},(1 \leqslant i, j \leqslant 2),
$$

$c_{i j}^{k}$ and $\Gamma_{i j}^{k}$ are the coefficients of the second fundamental form and Christoffel symbols, respectively [13]. The equation (2.1) is said to be Gaussian equation.

Let $\left\{N_{1}, N_{2}, \ldots, N_{n-2}\right\}$ be orthogonal frame field of $T_{P}^{\perp} M$, then shape operator on $M$ is defined by

$$
A: \chi^{\perp}(M) \times \chi(M) \rightarrow \chi(M), A_{N_{i}} X=-\left(\widetilde{\nabla}_{X_{i}} N_{i}\right)^{T},\left(X_{i} \in \chi(M)\right) .
$$

This operator is well defined, self adjoint and bilinear. It is well-known that

$$
\left\langle A_{N_{k}} X_{j}, X_{i}\right\rangle=\left\langle h\left(X_{i}, X_{j}\right), N_{k}\right\rangle=c_{i j}^{k},(1 \leqslant i, j \leqslant 2,1 \leqslant k \leqslant n-2) .
$$

Furthermore, the mean curvature vector of surfaces $M$ with the parametric $X(u, v)$ is given by ([8])

$$
\vec{H}=\frac{1}{2 W^{2}} \sum_{k=1}^{n-2}\left(c_{11}^{k} G+c_{22}^{k} E-2 c_{12}^{k} F\right) N_{k} .
$$

Definition 2.1. A surface in $\mathbb{E}^{n}$ is self-similar if

$$
\overrightarrow{\mathrm{H}}+\lambda X^{\perp}=0,
$$

where $\vec{H}$ is the mean curvature and $X^{\perp}$ stands for the projection of the position vector $X$ onto the normal space, respectively $[2,4]$. 
From the definition, a surface in $\mathbb{E}^{n}$ is self-similar if ([9])

$$
\left\langle\mathrm{H}, \mathrm{N}_{\mathrm{i}}\right\rangle+\lambda\left\langle\mathrm{X}, \mathrm{N}_{\mathrm{i}}\right\rangle=0, \quad \mathrm{i}=1,2,
$$

Thus from equation (2.2), we get

$$
\left\langle X_{u u}, N_{i}\right\rangle G+\left\langle X_{v v}, N_{i}\right\rangle E-2\left\langle X_{u v}, N_{i}\right\rangle F=-2 \lambda\left(E G-F^{2}\right)\left\langle X, N_{i}\right\rangle
$$

and

$$
c_{11}^{1} G+c_{22}^{1} E-2 c_{12}^{1} F=-2 \lambda\left(E G-F^{2}\right)\left\langle X, N_{1}\right\rangle, \quad c_{11}^{2} G+c_{22}^{2} E-2 c_{12}^{2} F=-2 \lambda\left(E G-F^{2}\right)\left\langle X, N_{2}\right\rangle .
$$

If a regular surface $X(u, v)$ in $\mathbb{E}^{4}$ satisfy above equalities, then $X(u, v)$ is self-similar surface.

Definition 2.2. Let $\gamma: I \subset \mathbb{R} \longrightarrow \mathbb{E}^{4}$ be a 4-ranked curve. A surface given with regular patch $X$ : $\mathrm{u} \subset \mathbb{E}^{2} \longrightarrow \mathbb{E}^{4}, X(u, v)=\gamma(u)+A(v) V_{2}(u)+B(v) V_{4}(u), v \in J \subset \mathbb{R}, 0 \leqslant u \leqslant 2 \pi$ is called generalized rotation surface if the following conditions satisfy:

$$
A^{\prime}(v)^{2}+B^{\prime}(v)^{2}>0, \quad\left(k_{1} A(v)-1\right)^{2}+\left(k_{2} A(v)-k_{3} B(v)\right)^{2}>0, v \in \mathbb{R},
$$

where $A(v), B(v)$ are differentiable functions [18]. A generalized rotation surface is defined as 1-parameter family of planar curve, lying in the normal plane spanned by $\left\{\mathrm{V}_{2}(\mathrm{u}), \mathrm{V}_{4}(\mathrm{u})\right\}$ vectors of the curve $\gamma$.

The parametric equation of the generalized rotation surfaces is given by

$$
X(u, v)=(f(v) \cos c u, f(v) \sin c u, g(v) \cos d u, g(v) \sin d u)
$$

where

$$
f(v)=\left(a+\frac{1}{k_{1}}\left(-a c^{2} A(v)+b d^{2} B(v)\right)\right), \quad g(v)=\left(b+\frac{1}{k_{1}}\left(-b d^{2} A(v)-a c^{2} B(v)\right)\right),
$$

$a, b, c, d \in \mathbb{R}[10]$.

For special values of $a, b, c, d$, we have special surfaces which are defined as follows.

\section{Definition 2.3.}

1. If we take $f(v)=\cos v, g(v)=\sin v, c \in \mathbb{R}^{+}$, and $d=1$, then the generalized rotation surfaces is called Lawson surface [16].

2. If we take $f(v)=a \cos v, g(v)=b \sin v, a, b \in \mathbb{R}$, and $c=d=1$, then the generalized rotation surface is called Aminov rotation surface [1].

3. If we take $f(v)=r(v) \cos v, g(v)=r(v) \sin v$, and $c=d=1$, then the generalized rotation surface is called Vranceanu surface [20].

4. If we take $f(v)=\cos v, g(v)=2 \sin v, c=1$, and $d=\frac{1}{2}$, then the generalized rotation surface is called flat Klein bottle [19].

5. If we take $f(v)=\cos ^{2} v, g(v)=\sin 2 v, c=2$, and $d=1$, then the generalized rotation surface is called Banchoff surface [6].

6. If we take $f(v)=v, g(v)=1, c=1$, and $d \in \mathbb{R}^{+}$, then the generalized rotation surface is called Loop surface [12].

\section{Self-similar generalized rotation surfaces in 4-dimensional Euclidean space}

In this section, we give necessary and sufficient conditions for a generalized rotation surface to be self-similar. Besides, we compute the conditions that Lawson surfaces, Aminow surfaces and Vranceanu surfaces to be self-similar under these conditions. Finally, we obtain that flat Klein Bottle, Banchoff and Loop surfaces are not self-similar. 
Theorem 3.1. A generalized rotation surface given by (2.4) is self-similar surface if and only if

$$
\frac{c^{2} f(v) g^{\prime}(v)-d^{2} f^{\prime}(v) g(v)}{(c f(v))^{2}+(d g(v))^{2}}+\frac{f^{\prime}(v) g^{\prime \prime}(v)-f^{\prime \prime}(v) g^{\prime}(v)}{\left(f^{\prime}(v)\right)^{2}+\left(g^{\prime}(v)\right)^{2}}+2 \lambda\left(f^{\prime}(v) g(v)-f(v) g^{\prime}(v)\right)=0 .
$$

Proof. Let $M$ be a generalized rotation surface. Then we get first and second partial derivatives of the surface $X(u, v)$ in $(2.4)$ :

$$
\begin{aligned}
\frac{\partial X}{\partial u} & =X_{u}(u, v)=(-c f(v) \sin c u, c f(v) \cos c u,-d g(v) \sin d u, d g(v) \cos d u), \\
\frac{\partial X}{\partial v} & =X_{v}(u, v)=\left(f^{\prime}(v) \cos c u, f^{\prime}(v) \sin c u, g^{\prime}(v) \cos d u, g^{\prime}(v) \sin d u\right), \\
X_{u u}(u, v) & =\left(-c^{2} f(v) \cos c u,-c^{2} f(v) \sin c u,-d^{2} g(v) \cos d u,-d^{2} g(v) \sin d u\right), \\
X_{u v}(u, v) & =\left(-c f^{\prime}(v) \sin c u, c f^{\prime}(v) \cos c u,-d g^{\prime}(v) \sin d u, d g^{\prime}(v) \cos d u\right), \\
X_{v v}(u, v) & =\left(f^{\prime \prime}(v) \cos c u, f^{\prime \prime}(v) \sin c u, g^{\prime \prime}(v) \cos d u, g^{\prime \prime}(v) \sin d u\right) .
\end{aligned}
$$

Also the normal vector to $M$ is obtained as

$$
\begin{aligned}
& \mathrm{N}_{1}=\frac{1}{\sqrt{\left(f^{\prime}(v)\right)^{2}+\left(g^{\prime}(v)\right)^{2}}}\left(-g^{\prime}(v) \cos c u,-g^{\prime}(v) \sin c u, f^{\prime}(v) \cos d u, f^{\prime}(v) \sin d u\right), \\
& N_{2}=\frac{1}{\sqrt{(\operatorname{cf}(v))^{2}(d g(v))^{2}}}(-d g(v) \sin c u, d g(v) \cos c u, c f(v) \sin d u,-c f(v) \cos d u) .
\end{aligned}
$$

Let we recall the coefficients of first and second fundamental form of a generalized rotation surface $X(u, v)$ [5]:

$$
\begin{aligned}
E & =\left\langle X_{u}, X_{u}\right\rangle=(c f(v))^{2}+(d g(v))^{2}, \\
F & =\left\langle X_{u}, X_{v}\right\rangle=0, \\
G & =\left\langle X_{v} X_{v}\right\rangle=\left(f^{\prime}(v)\right)^{2}+\left(g^{\prime}(v)\right)^{2}, \\
c_{11}^{1} & =\left\langle X_{u u}, N 1\right\rangle=\frac{c^{2} f(v) g^{\prime}(v)-d^{2} f^{\prime}(v) g(v)}{\sqrt{\left(f^{\prime}(v)\right)^{2}+\left(g^{\prime}(v)\right)^{2}}}, \\
c_{12}^{1} & =\left\langle X_{u v}, N_{1}\right\rangle=0, \\
c_{22}^{1} & =\left\langle X_{v v}, N_{1}\right\rangle=\frac{f^{\prime}(v) g^{\prime \prime}(v)-f^{\prime \prime}(v) g^{\prime}(v)}{\sqrt{\left(f^{\prime}(v)\right)^{2}+\left(g^{\prime}(v)\right)^{2}}} \\
c_{11}^{2} & =\left\langle X_{u u}, N_{2}\right\rangle=0, \\
c_{12}^{2} & =\left\langle X_{u v}, N_{2}\right\rangle=\frac{c d\left(f^{\prime}(v) g(v)-f(v) g^{\prime}(v)\right)}{\sqrt{(c f(v))^{2}+(d g(v))^{2}}}, \\
c_{22}^{2} & =\left\langle X_{v v}, N_{2}\right\rangle=0 .
\end{aligned}
$$

Therefore by using (2.3), (3.2), and (3.3), (3.1) is obtained.

So, from Theorem 3.1, we have following corollaries.

Corollary 3.2. For $\lambda=1$, the Lawson surface in $\mathbb{E}^{4}$ is a self-similar surface.

Proof. Substituting equations $\mathrm{f}(v)=\cos v, \mathrm{~g}(v)=\sin v, \mathrm{c} \in \mathrm{R}^{+}$, and $\mathrm{d}=1$ in equation (3.1) we have

$$
\frac{\mathrm{c}^{2} \cos v \cdot(\sin v)^{\prime}-(\cos v)^{\prime} \cdot \sin v}{\mathrm{c}^{2} \cos ^{2} v+\sin ^{2} v}+\frac{(\cos v)^{\prime}(\sin v)^{\prime \prime}-(\cos v)^{\prime \prime}(\sin v)^{\prime}}{\left((\cos v)^{\prime}\right)^{2}+\left((\sin v)^{\prime}\right)^{2}}=2 \lambda
$$

By $\lambda=1$ the above equation give self-similarity. 
Corollary 3.3. For $\lambda=\frac{1}{\mathrm{a}^{2}}=\frac{1}{\mathrm{~b}^{2}}$, the Aminov rotation surface in $\mathbb{E}^{4}$ is a self-similar surface.

Proof. Substituting equations $f(v)=a \cos v, g(v)=b \sin v, a, b \in R$, and $c=d=1$ in equation (3.1), we have

$$
\frac{a \cos v \cdot b \cos v+a \sin v \cdot b \sin v}{a^{2} \cos ^{2} v+b^{2} \sin ^{2} v}+\frac{a \cos v \cdot b \cos v+a \sin v \cdot b \sin v}{b^{2} \cos ^{2} v+a^{2} \sin ^{2} v}+2 \lambda\left(-a b \sin ^{2} v-a b \cos ^{2} v\right)=0
$$

or equal to

$$
2 \lambda=\frac{1}{a^{2} \cos ^{2} v+b^{2} \sin ^{2} v}+\frac{1}{b^{2} \cos ^{2} v+a^{2} \sin ^{2} v} .
$$

If we take $\lambda=\frac{1}{a^{2}}=\frac{1}{b^{2}}$, the proof is complete.

Corollary 3.4. For

$$
\left(\frac{1}{r^{2}(v)}+\frac{2\left(r^{\prime}(v)\right)^{2}-r(v) r^{\prime \prime}(v)+r^{2}(v)}{\left(\left(r^{\prime}(v)\right)^{2}+(r(v))^{2}\right) r^{2}(v)}\right)^{\prime}=0
$$

the Vranceanu surface in $\mathbb{E}^{4}$ is a self-similar surface.

Proof. Substituting equations $\mathrm{f}(v)=\mathrm{r}(v) \cos v, \mathrm{~g}(v)=\mathrm{r}(v) \sin v$ and $c=\mathrm{d}=1$ in equation (3.1), we have

$$
\frac{1}{r^{2}(v)}\left(1+\frac{2\left(r^{\prime}(v)\right)^{2}-r(v) r^{\prime \prime}(v)+r^{2}(v)}{\left(\left(r^{\prime}(v)\right)^{2}+(r(v))^{2}\right)}\right)=2 \lambda .
$$

If we take the derivative of this equality, it is concluded that $\lambda$ is fixed. This is the desired result.

Corollary 3.5. Flat Klein bottle in $\mathbb{E}^{4}$ is not a self-similar surface.

Proof. Substituting equations $\mathrm{f}(v)=\cos v, \mathrm{~g}(v)=2 \sin v, \mathrm{c}=1$ and $\mathrm{d}=\frac{1}{2}$ in equation (3.1), we have

$$
\left(\frac{9 \cos ^{4} v+3}{6 \cos ^{2} v+2}+1\right)=4 \lambda
$$

Since the $\lambda$ is dependent on $v$, flat Klein bottle in $\mathbb{E}^{4}$ is not a self-similar surface.

Corollary 3.6. Banchoff surface in $\mathbb{E}^{4}$ is not a self-similar surface.

Proof. Substituting equations $\mathrm{f}(v)=\cos ^{2} v, \mathrm{~g}(v)=\sin 2 v, \mathrm{c}=2$, and $\mathrm{d}=1$ in equation (3.1), we have

$$
\left(\frac{8 \cos ^{2} v \cos 2 v+\sin ^{2} 2 v}{4 \cos ^{4} v+\sin ^{2} 2 v}+\frac{4 \sin ^{2} 2 v+4 \cos ^{2} 2 v}{1+3 \cos ^{2} 2 v}+2 \lambda\left(\sin ^{2} 2 v-2 \cos ^{2} v \cos 2 v\right)\right)=0 .
$$

Since the $\lambda$ is dependent on $v$, Banchoff surface in $\mathbb{E}^{4}$ is not a self-similar surface.

Corollary 3.7. Loop surface in $\mathbb{E}^{4}$ is not a self-similar surface.

Proof. Substituting equations $\mathrm{f}(v)=v, \mathrm{~g}(v)=1, \mathrm{c}=1$, and $\mathrm{d} \in \mathrm{R}^{+}$in equation (3.1), we have

$$
\left(\frac{-d^{2}}{d^{2}+v^{2}}+2 \lambda\right)=0
$$

Since the $\lambda$ is dependent on $v$ and $d$, the Loop surface in $\mathbb{E}^{4}$ is not a self-similar surface.

\section{Self-similar tensor product and meridian surfaces in 4-dimensional euclidean space}

In this section, we examine the self-similarity of tensor product surfaces and meridian surfaces. We give necessary and sufficient conditions for to be self-similar surface of these surfaces. So, we can give 
the following definition.

Definition 4.1. Let $c_{1}: \mathbb{R} \rightarrow \mathbb{E}^{2}, c_{2}: \mathbb{R} \rightarrow \mathbb{E}^{2}$ be planar curves and

$$
c_{1}(u)=(\gamma(u), \delta(u)), c_{2}(v)=(\alpha(v), \beta(v)) .
$$

Then

$$
X(u, v)=(\alpha(v) \gamma(u), \beta(v) \gamma(u), \alpha(v) \delta(u), \beta(v) \delta(u))
$$

is called tensor product surfaces. Here the tensor product of the $c_{1}(u)$ and $c_{2}(v)$ curves means that $X=c_{1}(u) \otimes c_{2}(v)[17]$.

If we take $c_{1}(u)=(\cos u, \sin u), c_{2}(v)=(\alpha(v), \beta(v))$, then the tensor product surface becomes as

$$
M: X(u, v)=(\alpha(v) \cos u, \beta(v) \cos u, \alpha(v) \sin u, \beta(v) \sin u) \text {. }
$$

So, we have

Proposition 4.2. Tensor product surface (4.1) in $\mathbb{E}^{4}$ is self-similar surface if and only if

$$
\frac{\alpha(v) \beta^{\prime}(v)-\alpha^{\prime}(v) \beta(v)}{(\alpha(v))^{2}+(\beta(v))^{2}}+\frac{\alpha^{\prime}(v) \beta^{\prime \prime}(v)-\alpha^{\prime \prime}(v) \beta^{\prime}(v)}{\left(\alpha^{\prime}(v)\right)^{2}+\left(\beta^{\prime}(v)\right)^{2}}+2 \lambda\left(\alpha^{\prime}(v) \beta(v)-\alpha(v) \beta^{\prime}(v)\right)=0 .
$$

Proof. The first and second partial derivatives and normal vectors of the surface $X(u, v)$ in $(4.1)$ are given as

$$
\begin{aligned}
\frac{\partial X}{\partial u} & =X_{u}(u, v)=(-\alpha(v) \sin u,-\beta(v) \sin u, \alpha(v) \cos u, \beta(v) \cos u), \\
\frac{\partial X}{\partial v} & =X_{v}(u, v)=\left(\alpha^{\prime}(v) \cos u, \beta^{\prime}(v) \cos u, \alpha^{\prime}(v) \sin u, \beta^{\prime}(v) \sin u\right), \\
X_{u u}(u, v) & =(-\alpha(v) \cos u,-\beta(v) \cos u,-\alpha(v) \sin u,-\beta(v) \sin u), \\
X_{v u}(u, v) & =\left(-\alpha^{\prime}(v) \sin u,-\beta^{\prime}(v) \sin u, \alpha^{\prime}(v) \cos u, \beta^{\prime}(v) \cos u\right), \\
X_{v v}(u, v) & =\left(\alpha^{\prime \prime}(v) \cos u, \beta^{\prime \prime}(v) \cos u, \alpha^{\prime \prime}(v) \sin u, \beta^{\prime \prime}(v) \sin u\right), \\
N_{1} & =\frac{1}{\left\|c_{2}^{\prime}\right\|}\left(-\beta^{\prime}(v) \cos u, \alpha^{\prime}(v) \cos u,-\beta^{\prime}(v) \sin u, \alpha^{\prime}(v) \sin u\right), \\
N_{2} & =\frac{1}{\left\|c_{2}\right\|}(-\beta(v) \sin u, \alpha(v) \sin u, \beta(v) \cos u,-\alpha(v) \cos u) .
\end{aligned}
$$

From [7] coefficients of first and second fundamental form of surface $X(u, v)$ in (4.1) are given as follows

$$
\begin{aligned}
E & =\left\langle X_{\mathfrak{u}}, X_{\mathfrak{u}}\right\rangle=(\alpha(v))^{2}+(\beta(v))^{2}, \\
F & =\left\langle X_{u}, X_{v}\right\rangle=0, \\
G & =\left\langle X_{v}, X_{v}\right\rangle=\left(\alpha^{\prime}(v)\right)^{2}+\left(\beta^{\prime}(v)\right)^{2}, \\
c_{11}^{1} & =\left\langle X_{u u}, N 1\right\rangle=\frac{\alpha(v) \beta^{\prime}(v)-\alpha^{\prime}(v) \beta(v)}{\left\|c_{2}^{\prime}\right\|}, \\
c_{12}^{1} & =\left\langle X_{u v}, N_{1}\right\rangle=0, \\
c_{22}^{1} & =\left\langle X_{v v}, N_{1}\right\rangle=\frac{\alpha^{\prime}(v) \beta^{\prime \prime}(v)-\alpha^{\prime \prime}(v) \beta^{\prime}(v)}{\left\|c_{2}^{\prime}\right\|}, \\
c_{11}^{2} & =\left\langle X_{u u}, N_{2}\right\rangle=0, \\
c_{12}^{2} & =\left\langle X_{u v}, N_{2}\right\rangle=\frac{\alpha^{\prime}(v) \beta(v)-\alpha(v) \beta^{\prime}(v)}{\left\|c_{2}\right\|} \\
c_{22}^{2} & =\left\langle X_{v v}, N_{2}\right\rangle=0 .
\end{aligned}
$$

If the equalities (4.2) and (4.3) are used in equation (2.3), then the desired result is obtained.

Finally, we examine necessary and sufficient condition to be self-similar surface of meridian surfaces. So, we can give the following definition. 
Definition 4.3. Let $\left\{e_{1}, e_{2}, e_{3}, e_{4}\right\}$ be standard orthogonal frame in $\mathbb{E}^{4}, \alpha: \alpha(u)=\left(\alpha_{1}(u), \alpha_{2}(u)\right)$ and $\gamma$ : $\gamma(v)=\left(\gamma_{1}(v), \gamma_{2}(v), \gamma_{3}(v)\right)$ be unit speed curves. Frame of curve $\gamma(v)$ is $\{\mathbf{T}(v), \mathbf{N}(v), \gamma(v)\}$, while $\mathbf{T}=\gamma^{\prime}$, provides following equations

$$
\gamma^{\prime}(v)=\mathrm{T}(v), \quad \mathrm{T}^{\prime}(v)=\mathrm{k}(v) \mathrm{N}(v)-\gamma(v), \quad \mathrm{N}^{\prime}(v)=-\mathrm{k}(v) \mathrm{T}(v),
$$

where curvature of curve $\gamma(v)$ is $k(v)$ and curvature of $\alpha(u)$ is $\kappa_{\alpha}(u)$.

It is known that [11], meridian surface is parameterized by

$$
M: X(u, v)=\alpha_{1}(u) \gamma(v)+\alpha_{2}(u) e_{4} .
$$

So, we have the following proposition.

Proposition 4.4. Meridian surface (4.4) in $\mathbb{E}^{4}$ is self-similar surface if and only if

$$
\alpha_{1}(u) \kappa(v)=0
$$

and

$$
\kappa_{\alpha}(u) \alpha_{1}(u)+\alpha_{2}^{\prime}(u)+2 \lambda \alpha_{1}(u)\left(\alpha_{1}^{\prime}(u) \alpha_{2}(u)-\alpha_{1}(u) \alpha_{2}^{\prime}(u)\right) .
$$

Proof. The first and second partial derivatives and normal vectors of the surface $X(u, v)$ are given as

$$
\begin{aligned}
\mathrm{X}_{\mathrm{u}}(\mathrm{u}, v) & =\alpha_{1}^{\prime}(\mathrm{u}) \gamma(v)+\alpha_{2}^{\prime}(\mathrm{u}) e_{4}, \\
\mathrm{X}_{v}(\mathrm{u}, v) & =\alpha_{1}(\mathrm{u}) \mathrm{T}(v), \\
\mathrm{X}_{\mathrm{uu}}(\mathrm{u}, v) & =\alpha_{1}^{\prime \prime}(\mathrm{u}) \gamma(v)+\alpha_{2}^{\prime \prime}(\mathrm{u}) e_{4}, \\
\mathrm{X}_{v \mathrm{u}}(\mathrm{u}, v) & =\alpha_{1}^{\prime}(\mathrm{u}) \mathrm{T}(v), \\
\mathrm{X}_{v v}(\mathrm{u}, v) & =\alpha_{1}(\mathrm{u}) \kappa(v) \mathrm{N}(v)-\alpha_{1}(\mathrm{u}) \gamma(v), \\
\mathrm{N}_{1} & =\mathrm{N}(v), \\
\mathrm{N}_{2} & =-\alpha_{2}^{\prime}(\mathrm{u}) \gamma(v)+\alpha_{1}^{\prime}(\mathrm{u}) e_{4} .
\end{aligned}
$$

Also, coefficients of first and second fundamental form of surface $X(u, v)$ are given as follows [7]

$$
\begin{aligned}
E & =\left\langle X_{u}, X_{u}\right\rangle=1, \\
F & =\left\langle X_{u}, X_{v}\right\rangle=0, \\
G & =\left\langle X_{v}, X_{v}\right\rangle=\left(\alpha_{1}(u)\right)^{2}, \\
c_{11}^{1} & =\left\langle X_{u u}, N 1\right\rangle=0, \\
c_{12}^{1} & =\left\langle X_{u v}, N_{1}\right\rangle=0, \\
c_{22}^{1} & =\left\langle X_{v v}, N_{1}\right\rangle=\alpha_{1}(u) \kappa(v), \\
c_{11}^{2} & =\left\langle X_{u u}, N_{2}\right\rangle=0, \\
c_{11}^{2} & =\left\langle X_{u u}, N_{2}\right\rangle=\kappa_{\alpha}(u), \\
c_{12}^{2} & =\left\langle X_{u u}, N_{2}\right\rangle=0, \\
c_{22}^{2} & =\left\langle X_{v v}, N_{2}\right\rangle=\alpha_{1}(u) \alpha_{2}^{\prime}(u) .
\end{aligned}
$$

If the equalities (4.5) and (4.6) are used in equation (2.3), then the desired result is obtained.

\section{References}

[1] Y. A. Aminov, Surfaces in $E^{4}$ with a Gaussian curvature coinciding with a Gaussian torsion up to the sign, Math. Notes, 56 (1994), 1211-1215. 1, 2

[2] H. Anciaux, Construction of Lagrangian Self-similar Solutions to the Mean Curvature Flow in $C^{n}$, Geom. Dedicata, 120 (2006), 37-48. 1, 2.1 
[3] H. Anciaux, Two non existence results for the self-similar equation in Euclidean 3-space, J. Geom., 96 (2009), 1-10.

[4] H. Anciaux, P. Romon, Cyclic and ruled Lagrangian surfaces in Euclidean four space, Bull. Braz. Math. Soc. (N.S.), 40 (2009), 341-369. 1, 2.1

[5] K. Arslan, B. Bayram, B. Bulca, G. Öztürk, Generalized Rotation Surfaces in E ${ }^{4}$, Results Math., 61 (2012), 315-327. 3

[6] T. F. Banhoff, Computer Animation and the Geometry of Surfaces in 3 and 4-Space, Proceedings of the International Congress of Mathematicians (Helsinki), 1978 (1978), 1005-1013. 1, 5

[7] B. Bulca, $E^{4}$ deki Yüzeylerin Bir Karekterizasyonu, Doktora tezi, Bursa, (2012). 4, 4

[8] B.-Y. Chen, Geometry of submanifolds, Marcel Dekker, New York, (1973). 2

[9] E. Etemoğlu, K. Arslan, B. Bulca, Self similar surfaces in Euclidean space, Selçuk J. Appl. Math., 14 (2013), 71-81. 1, 2.1

[10] G. Ganchev, V. Milousheva, On the Theory of Surfaces in the Four Dimensional Euclidean Space, Kodai Math. J., 31 (2008), 183-192. 1, 2

[11] G. Ganchev, V. Milousheva, Invariants and Bonnet-type therom for Surfaces in R ${ }^{4}$, Cent. Eur. J. Math., 8 (2010), $993-$ 1008. 1,4

[12] L. Geysens, P. Verheyen, L. Verstraelen, Characterization and Examples of Chen submanifolds, J. Geom., 20 (1983), 47-62. 1,6

[13] A. Gray, Modern Differential Geometry of Curves and Surfaces, CRS Press, Boca Raton, (1993). iii, 2

[14] K. Hanbay, N. Alpaslan, M. F. Talu, D. Hanbay, Principal curvatures based rotation invariant algorithms for efficient texture classification, Neurocomputing, 199 (2016), 77-89. 1

[15] K. Hanbay, M. F. Talu, A novel active contour model for medical images via the Hessian matrix and eigenvalues, Comput. Math. Appl., 75 (2018), 3081-3104. 1

[16] H. B. Lawson, Complete Minimal Surfaces in $S^{3}$, Ann. of Math. (2), 92 (1970), 335-374. 1, 1

[17] I. Mihai, B. Rouxel, Tensör product surfaces of Euclidean plane curves, Rend. Sem. Mat. Messina, 27 (1995), $308-315$. $1,4.1$

[18] C. L. E. Moore, Surfaces of Rotations in a Space of Four Dimensions, Ann. of Math. (2), 21 (1919), 81-93. 2.2

[19] C. Tompkins, A flat Klein bottle isometrically embedded in Euclidean 4-space, Bull. Amer. Math. Soc., 47 (1941), 508-519. 1,4

[20] G. Vranceanu, Surfaces de Rotation dans E ${ }^{4}$, (French) Rev. Roumaine Math. Pures Appl., 22 (1977), 857-862. 1, 3 